\title{
COMMENTARY
}

\section{Nanobiophotonics: example of scientific convergence}

\author{
Anderson S. L. Gomes \\ Department of Physics, Universidade Federal de Pernambuco, Recife, 50670-901, PE, \\ Brazil \\ anderson@df.ufpe.br
}

In a world where convergence is becoming the new wave, nanobiophotonics sets the frame as an outstanding example, as it is at the focal point of three well-established fields: nanotechnology, photonics, and biomedical sciences. The fast development of nanotechnology, from the 1980's, supported by tools that can observe and manipulate in the nanoworld has found in photonics - defined as the technology of generating, harnessing and transmitting photons - $\mathrm{a}$ special companion for applications. Nanophotonics is already a discipline in its own right [1]. Recent developments in nanotechnology and photonics related to biomedical sciences, both basic and applied, have led to the newly born field of nanobiophotonics. Figure 1 depicts this convergence. Notice in this figure the side roads that exist, leading to other cross-disciplinary and fertile fields, such as nanophotonics, nanomedicine and biophotonics.

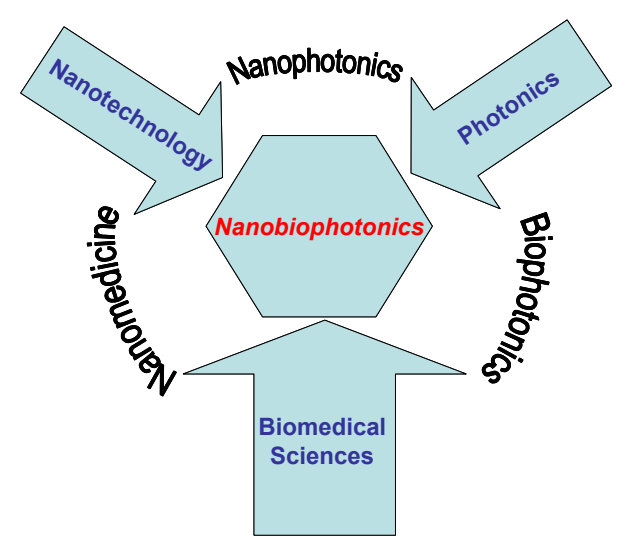

Fig.1. Pictorial description of the scientific convergence leading to Nanobiophotonics.

Advances in nanobiophotonics are striking. Leading experts worldwide have put together multidisciplinary teams to address challenges that range from noninvasive imaging to therapies and treatment, all using photons and nanoparticles of different shapes and functionalities. This commentary focuses on two of these advances.

Far-field Optical Nanoscopy. This emerging field has pushed optical microscopy to the nanoscale, providing the ability to optically observe well beyond the diffraction limit. Its main advantage for nanobiophotonics, as opposed to near-field microscopy, is the ability to see inside biological media, such as cells [2]. Several approaches have been developed to break the diffraction limit, such as optical confocal microscopy, multiphoton microscopy, and 4Pi microscopy (mainly two-photon excited). Perhaps the most exciting advances have arisen from the pioneering work of Hell and co-workers who introduced the stimulated emission depletion (STED) microscopy, providing 
resolutions of $\sim 20 \mathrm{~nm}$. The STED approach is based on photo-switching capabilities of transition states of fluorophores to effectively reduce the size of the focused spotpotentially all the way down to a single molecule. As summarized in Ref. 3, in a STED microscope, "the focal spot is overlapped with a ring of light that switches off fluorescence everywhere in the spot except at the centre of the ring. The outcome is a much smaller fluorescence spot that, when scanned across the specimen, gives superresolution images automatically". More recent approaches, broadly based on photoswitching mechanisms, have been used for detecting single molecule in cells $[2,3]$. Most certainly, visualization by photonic methods will impact developments in nanobiophotonics.

Exploiting Nanoparticles. Fluorescent emission is perhaps the best way to study and understand what is going on in biological media. Both academic and industrial researchers have used nanoparticles - dielectric and metallic - to target non-fluorescent materials as well as to enhance the fluorescence in already fluorescent media.

When using metallic nanoparticles, the well-known effect of plasmon resonance occurs [4]. Plasmons are collective oscillations of free electrons on metallic surfaces and in metallic particles. The plasmonic influence on its environment depends strongly on the nanoparticle size and shape. Figure 2 shows the selection of plasmon resonance by choosing the size and shape of a nanoparticle made of a particular metal or alloy [5]. By choosing an appropriate type of nanoparticle, many biological materials can be studied in the presence of such nanoobjects, for instance leading to the enhancement of fluorescence [6].

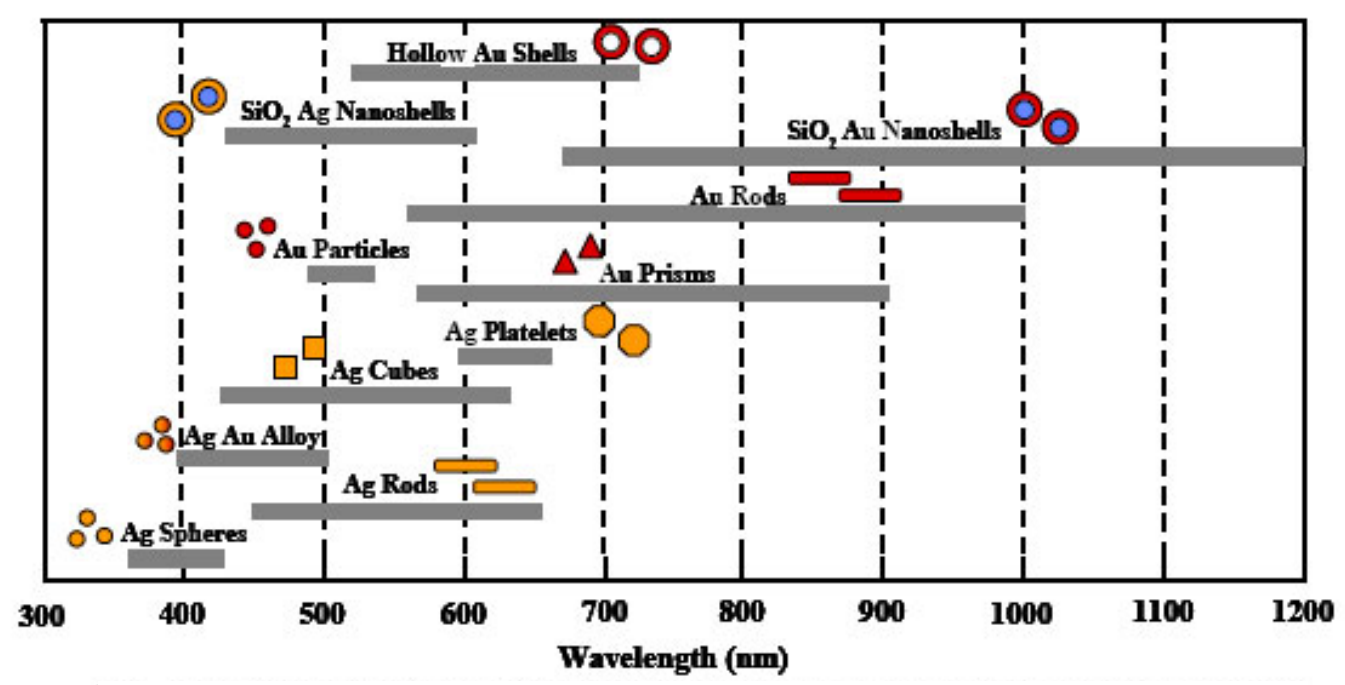

Fig. 2. Selection of plasmon resonance in metallic nanoparticles by choosing their size and shape and by forming composite structures, viz., alloys and nanoshells (from Ref. 5, with permission).

Besides metallic nanoparticles, semiconductor quantum dots (QDs) and nanophosphors containing rare-earth doped (RED) ions are among the family of nanomaterials used for bio-applications. QDs have been widely exploited for bioimaging as their color depends on size-dependent absorption, manifesting as a blue shift with the reduction of size. QDs offer advantages over organic dyes used as fluorescent markers. Among the advantages, one can highlight the narrow (less than $40 \mathrm{~nm}$ ) emission bandwidth as compared to dyes (wider than $80 \mathrm{~nm}$ ), and they do not bleach as easily as dyes. Because most of the QDs employed so far are made of CdSe, a concern about their toxicity has been raised. A way around this problem is to use QDs of materials such as InP, which are, however, more cumbersome to prepare. There have also been reports of 
new chemical routes to fabricate efficient QDs with low toxicity, as $\operatorname{InP} / \mathrm{ZnS}$, called coreshell QDs [1].

RED materials are quite well known and provide a myriad of applications. Nanophosphors containing RED ions have opened up a new avenue to high-contrast bioimaging, with the advantage of operating in the near-infrared (NIR, 700-1000 nm) range, which is the optical transmission window for biological media. As an example, $\mathrm{Tm}^{3+}$ - and $\mathrm{Yb}^{3+}$-doped fluoride nanophosphors have been prepared and tested for bioimaging and cell viability [7]. These nanophosphors exploits a NIR-NIR upconversion mechanism, well known in spectroscopy, to provide continuous-wave (cw) excitation in the NIR range for bioimaging. Very positive results have brought a new dimension to bioimaging [7], particularly because appropriate RED ions can be used in combination with magnetic resonance, thus allowing further insight into the biomaterial being studied.

Nanobiophotonics is already playing a major role in practice. The capability of optically observing the nanoworld, be it through light-matter interaction at the nanoscale or through optical nanoscopy, is available. A version of the STED microscope, according to Ref. 3, should be commercialized shortly. Imaging and therapy, using metal nanoparticles, are already playing an important role in cancer treatment and diagnostic. The work of the Halas group, from the late 1990's, exemplifies the achievements thus far. As reviewed in Ref. 8, metal nanoshells have enabled photonics-based imaging and cancer therapy. As can be seen from Fig. 2, by tuning the plasmon resonance in nanoshells (by precisely selecting relative dimensions of the core and the shell), the optical resonance can chosen to occur from the near-ultraviolet to the NIR range. Conjugation protocols used for spherical nanoparticles have been shown to apply to nanoshells [8]. Among the multitude of examples employing nanoshells, applications to diagnosis and therapy of colorectal cancer have been demonstrated [9]. As colorectal cancer is the third most common type of cancer in USA alone, this development is certainly an important breakthrough.

Another example demonstrates that silver nanoparticles in conjunction with nonlinear optics can be used for fluorescence enhancement in amino acids [10]. Tryptophan is the dominant source of UV absorption and emission in proteins. The intrinsic fluorescence emission of a cell is mostly due to excitation of tryptophan residues, with additional contributions of tyrosine and phenylalanine. As tryptophan is a relatively rare amino acid, many proteins contain only a small number of tryptophan residues. For many organisms, including humans, it is one of the essential amino acids, which cannot be synthesized by the human body and therefore must be part of the diet. Tryptophan is a precursor for serotonin, one of the key chemicals in the brain for regulation of the mood. A colloidal suspension of 9-nm tryptophan-silver particles in water has its fluorescence emission peak at $\sim 360 \mathrm{~nm}$. But it behaves quite differently when excited by a femtosecond quasi-cw $(76 \mathrm{MHz})$ source at $800-\mathrm{nm}$ wavelength [10]. Whereas quenching of fluorescence is observed for one- and two-photon excitation, an enhancement of fluorescence occurs upon three-photon excitation. This has been explained as due to the plasmon resonance in silver, which provides the pathway for a (2+1)-photon process for 800-nm excitation. In Fig. 3, the fluorescence quenching as a function of the volume fraction $f$ of silver in the colloid is shown for one- and two-photon excitations. The absence of silver nanoparticles is indicated by $f=0$ (maximum fluorescent emission). In the same figure, the enhancement of fluorescence for the three-photon excitation is shown as $f$ increases, and is normalized such that the fluorescence is minimum when $f=0$. The saturation behavior is not yet understood. This three-photon fluorescence enhancement is extremely useful for bioimaging, as it was also observed using a wide-field microscope for imaging the three-photon-excited fluorescence. 


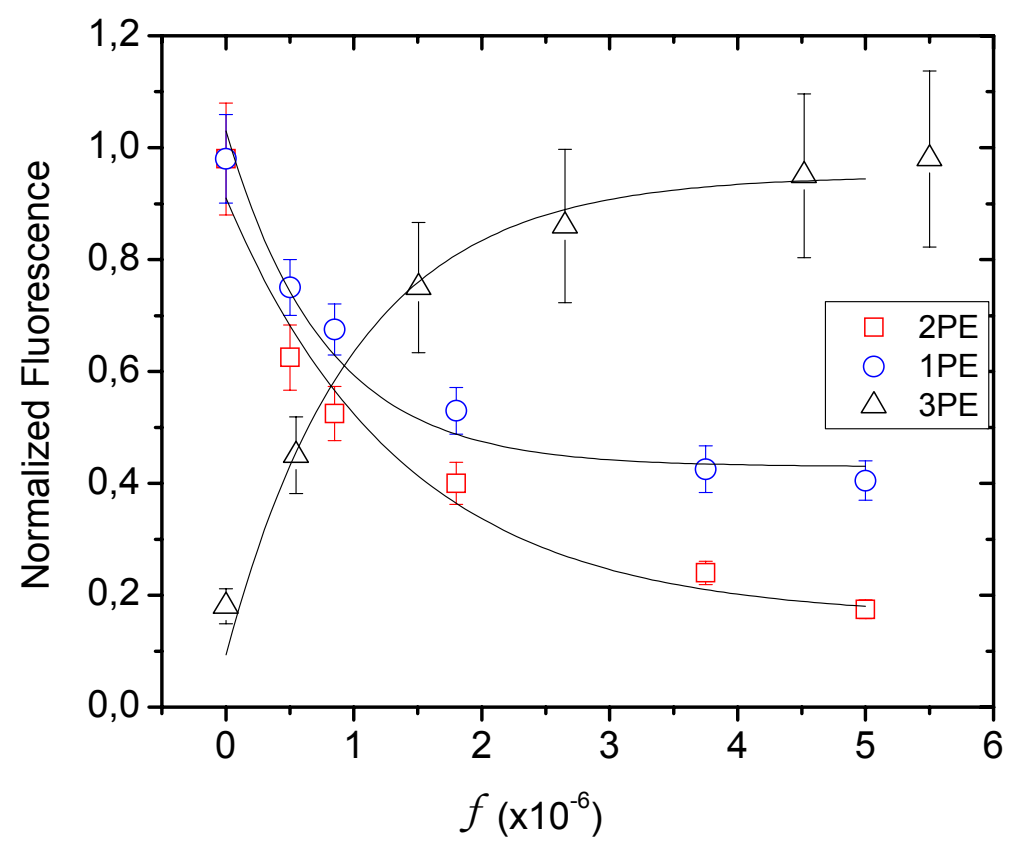

Fig. 3. Normalized fluorescence (at $360 \mathrm{~nm}$ ) peak intensity of tryptophan-silver colloids as a function of the volume fraction of silver nanoparticles, excited by one- (1PE, circles), two- (2PE, squares) and three-photon (3PE, triangles) excitation at $800-\mathrm{nm}$ excitation wavelength.

Thus, nanobiophotonics - the convergence of rapidly growing fields-will greatly affect the diagnoses and treatments of many diseases. It will also provide a better understanding of the bionanoworld. I have pointed out examples of recent advances in bioimaging, diagnostics and treatment using light and nanoparticles in ways never done before. Different nanomaterials - metals, dielectrics, and/or semiconductors - can be used if appropriately tailored and functionalized. Optical sources, either cw or pulsed, can be used to allow light-matter interaction at the nanoscale, thus revealing the bionanoworld.

\section{Acknowledgments}

A.S.L.G. gratefully acknowledges the support of CAPES, CNPq/MCT and FACEPE, through the Center of Excellence in Nano and Biophotonics, the Nanophotonics Network and the Photonics INCT.

\section{References}

[1] P. N. Prasad, Nanophotonics, Wiley, New York (2004). [doi:10.1002/0471670251].

[2] S. W. Hell, "Far-field optical nanoscopy," Science 316, 1153-1158 (2007) [doi: 10.1126/science.1137395].

[3] N. Blow, "New ways to see a smaller world," Nature 456, 825-828 (2008) [doi: $10.1038 / 456825 \mathrm{a}$ ].

[4] D. Felbacq, "Plasmons go quantum," J. Nanophoton. 2, 020302 (2008) [doi: $10.1117 / 1.2953244$ ].

[5] S. A. Kalele, N. R. Tiwari, S. W. Gosavi, and S. K. Kulkarni, "Plasmon-assisted photonics at the nanoscale," J. Nanophoton. 1, 012501 (2007) [doi: 10.1117/1.2748429]. 
[6] J. R. Lakowicz, "Plasmonics in biology and plasmon-controlled fluorescence," Plasmonics 1, 5-33 (2006) [doi: 10.1007/s11468-005-9002-3].

[7] M. Nyk, R. Kumar, T. Y. Ohulchanskyy, E. J. Bergey, and P. N. Prasad, "High contrast in vitro and in vivo photoluminescence bioimaging using near infrared to near infrared up-conversion in $\mathrm{Tm}^{3+}$ and $\mathrm{Yb}^{3+}$ doped fluoride nanophosphors," Nano Lett. 8, 3834-3838 (2008) [doi: 10.1021/nl802223f].

[8] C. Loo, A. Lin, L. Hirsch, M.-H. Lee, J. Barton, N. Halas, J. West, and R. Drezek, "Nanoshell-enabled photonics-based imaging and therapy of cancer," Technol. Cancer Res. Treat. 3, 33-40 (2004).

[9] P. Fortina, L. J. Kricka, D. J. Graves, J. Park, T. Hyslop, F. Tam, N. Halas, S. Surrey, and S. A. Waldman, "Applications of nanoparticles to diagnostics and therapeutics in colorectal cancer," Trends Biotechnol. 24, 145-152 (2007) [doi: 10.1016/j.tibtech.2007.02.005].

[10] D. Rativa, A. S. L. Gomes, S. Wachsmann-Hogiu, D. L. Farkas, and R. E. de Araujo, "Nonlinear excitation of tryptophan emission enhanced by silver nanoparticles," J. Fluoresc. 18, 1151-1155 (2008) [doi: 10.1007/s10895-0080366-6]. 